\title{
EGFR and $K-R A S$ mutations and ERCC1, TUBB3, TYMS, RRM1 and $E G F R$ mRNA expression in non-small cell lung cancer: Correlation with clinical response to gefitinib or chemotherapy
}

\author{
NANNAN GUO ${ }^{1,2^{*}}$, WEN ZHANG ${ }^{2 *}$, BAOSHI ZHANG ${ }^{2}$, YINGJIE LI $^{2}$, JIAN TANG $^{2}$, SHAOJUN LI $^{2}$, \\ YINGNAN ZHAO ${ }^{2}$, YUNLONG ZHAO ${ }^{2}, \mathrm{HUI} \mathrm{XIA}^{2}$ and CHANGHAI YU ${ }^{2}$ \\ ${ }^{1}$ Medical School of Chinese PLA General Hospital, Beijing 100853; ${ }^{2}$ Department of Thoracic-Cardio Surgery, \\ First Affiliated Hospital of PLA General Hospital, Beijing 100048, P.R. China
}

Received February 13, 2015; Accepted June 15, 2015

DOI: $10.3892 / \operatorname{mco} .2015 .611$

\begin{abstract}
Personalizing medicines has refined the traditional treatments for non-small-cell lung cancer (NSCLC). In the present study, efforts towards personalizing delivery of care based on the status of EGFR and $K-R A S$ mutations, and mRNA expression levels of ERCC1, TUBB3, TYMS, RRM1 and $E G F R$ by choosing appropriate treatments for 52 patients with NSCLC were discussed. Among these 52 NSCLC patients, there were 14 patients treated with gefitinib. Ten patients with EGFR exon 21 point mutations or exon 19 deletions had better treatment outcomes following gefitinib treatment $(71.4 \%)$. There were 38 patients treated with platinum-based chemotherapy. Docetaxel-platinum based chemotherapy was chosen as the first-line treatment when the patients had low or median ERCC1/TUBB3 expression and gemcitabine-platinum based chemotherapy was chosen when the patients had low or median ERCC1/RRM1 expression. In total, 26 cases had mRNA expression levels of ERCC1/TUBB3 or ERCC1/RRMI that could be used to predict the treatment outcomes of chemotherapy (68.4\%). The present results indicated that the mutation status of $E G F R$, as well as the mRNA expression levels of $E R C C 1, T U B B 3$ and $R R M 1$, could be used as predictors of the response to gefitinib or chemotherapy.
\end{abstract}

Correspondence to: $\mathrm{Dr}$ Changhai Yu, Department of Thoracic-Cardio Surgery, First Affiliated Hospital of PLA General Hospital, 51 Fucheng Road, Beijing 100048, P.R. China

E-mail: yuch304@163.com

* Contributed equally

Key words: non-small-cell lung cancer, EGFR and K-RAS mutation status, ERCC1, TUBB3, RRM1 and EGFR mRNA expression levels, gefitinib, chemotherapy

\section{Introduction}

Lung cancer, of which nearly $80-85 \%$ is diagnosed as non-small-cell lung cancer (NSCLC), is one of the leading causes of fatality worldwide (1). The adjuvant chemotherapy, including the combinations of platinum and cytotoxic agents (such as docetaxel and gemcitabine) and epidermal growth factor receptor (EGFR)-targeted therapy, such as gefitinib (AstraZeneca, London, UK), has become common treatments for NSCLC (2). However, drug resistance causes unsatisfactory clinical responses to these medicines. Therefore, it is essential to understand the molecular markers associated with resistance to these medicines, to aid oncologists in choosing the more effective anti-tumor drugs for patients to achieve the most advantageous potential outcomes.

The major molecular markers associated with clinical response to gefitinib or chemotherapy in NSCLC include the status of EGFR, K-RAS mutations and mRNA expression levels of excision repair cross-complementing 1 (ERCC1), class III $\beta$-tubulin (TUBB3), thymidylate synthase (TYMS), ribonucleotide reductase subunit M1 (RRMI) and EGFR (3-14). EGFR is a member of the ErbB family of receptors. Mutations within the tyrosine kinase domain of EGFR account for increased sensitivity to the tyrosine kinase inhibitor (TKIs), such as gefitinib $(3,4)$. However, an insertion mutation and the point mutation T790M in exon 20 of EGFR are associated with resistance to TKIs (5). Previous studies have demonstrated that overexpression of EGFR was associated with increased sensitivity to gefitinib $(6,7)$. K-RAS encodes a small guanosine triphosphate (GTP)-binding protein involved in numerous cellular processes, including proliferation and apoptosis (8). The wild-type K-RAS protein has intrinsic GTPase activity that catalyzes the hydrolysis of bound GTP to GDP. Mutations within the K-RAS gene, $98 \%$ in codons 12,13 or 61 , result in locking of oncogenic K-RAS into the GTP-bound state and lead to the constitutive activation of RAS signaling. $K-R A S$ mutations are associated with resistance to gefitinib (9). ERCC1 is a DNA repair endonuclease responsible for the 5'-incision during DNA excision repair. Overexpression of ERCC1 is correlated to resistance to platinum-based chemotherapy (10). TUBB3 encodes a class III member of the $\beta$ tubulin protein family. Clinical studies have 
shown that high TUBB3 expression is associated with resistance to docetaxel and paclitaxel $(11,12)$. TYMS is the enzyme used for DNA synthesis and repair. The high TYMS expression is associated with resistance to fluorouracil in NSCLC (13). RRM1 is one of the subunits of ribonucleoside-diphosphate reductase, which is essential for the production of deoxyribonucleotides prior to DNA synthesis. The high RRM1 expression in NSCLC is connected with resistance to gemicitabin-based therapy (14).

In the present study, the mutation status of EGFR and $K$-RAS genes, as well as the mRNA expression levels of ERCCI, TUBB3, TYMS, RRMI and EGFR genes on the tumor tissue samples from 52 NSCLC patients were analyzed. The patients were treated with gefitinib or platinum-based chemotherapy according to the status of these molecular markers. The associations of the status of these molecular markers and corresponding clinical responses were evaluated to determine whether these biomarkers could be used as predictors of the response to gefitinib or chemotherapy.

\section{Materials and methods}

Patients and study design. Patients were recruited by the Beijing First Affiliated Hospital of PLA General Hospital (304 Hospital, Beijing, China) between January 2013 and June 2014. A total of 52 patients who underwent surgery for NSCLC were enrolled in the study. The patient clinical characteristics are listed in Table I. A total of 52 tissue samples were obtained following surgery. The tissue samples were fixed in $10 \%$ neutral formalin for $16 \mathrm{~h}$, desiccated and embedded in paraffin. The diagnosis of NSCLC was based on the cytological or histological findings and histological types were determined according to the World Health Organization criteria (15). For each formalin-fixed paraffin-embedded (FFPE) tissue sample, the tumor tissues had been cut by microdissection techniques and sent to the Guangzhou SurExam Medical Test Center for EGFR and K-RAS mutations, and ERCC1, TUBB3, TYMS, $R R M I$ and $E G F R$ mRNA expression analysis.

The NSCLC patients were administered with the platinum-based chemotherapy or gefitinib, according to the status of EGFR and $K-R A S$ mutations, and the mRNA expression levels of ERCC1, TUBB3, TYMS, RRM1 and EGFR. Computed tomography (CT) scans were performed at 4 weeks after treatments. The clinical responses to treatments were classified as progressive disease (PD), stable disease (SD), complete response (CR) and partial response (PR) according to the Response Evaluation Criteria in Solid Tumors criteria (15). The correlation of clinical responses and status of the biomarkers were analyzed. The study was approved by the Ethics Committee of the 304 Hospital. Written informed consent was obtained from all the patients enrolled.

$D N A$ extraction and mutation analysis of EGFR and $K-R A S$. The analysis of the EGFR and $K$-RAS mutation status was performed at SurExam Medical Test Center. The Maxwell ${ }^{\circledR}$ system (Promega Corp., Madison, WI, USA) was used to extract DNA from paraffin-embedded tissues. The status of EGFR mutations in exons 18, 19, 20 and 21 and $K$-RAS mutations in codon 12,13 and 61 was screened by SurPlex ${ }^{\circledR}$-xTAG70plex (SurExam Biotech Co Ltd. Guangzhou, Guangdong, China). The method includes five steps (16).
Table I. Patient clinical characteristics.

\begin{tabular}{lc}
\hline Variables & Patients \\
\hline Age, median years (range) & $59(39-78)$ \\
Gender, n (\%) & $38(73.1)$ \\
Male & $14(26.9)$ \\
Female & \\
Smoker, n (\%) & $33(63.4)$ \\
Yes & $19(36.6)$ \\
No & \\
Histology, n (\%) & $28(53.8)$ \\
Adenocarcinoma & $24(46.2)$ \\
Non-adenocarcinoma & \\
Differentiation, n (\%) & $31(59.6)$ \\
Well and moderate & $21(40.4)$ \\
Poor & \\
Stage, n (\%) & $15(28.8)$ \\
II & $28(53.8)$ \\
III &
\end{tabular}

ERCC1, TUBB3, TYMS, RRM1 and EGFR mRNA expression analysis. ERCC1, TUBB3, TYMS, RRM1 and EGFR mRNA expression analysis was performed by the multiplex branched-DNA technology at SurExam Medical Test Center. This technology includes a sandwich nucleic acid hybridization method in which mRNAs are captured through cooperative hybridization of multiple probes and subsequently coupled with a fluorescence signal amplification system (17). The signals were detected by the Luminex 200 system, as described previously (17).

Statistical analysis. The data were analyzed using SPSS 19.0 software package (IBM Corp., Armonk, NY, USA). The correlation between gene mutation status and mRNA expression levels was evaluated by Spearman correlation coefficients. $\mathrm{P}<0.05$ was considered to indicate a statistically significant difference.

\section{Results}

EGFR and K-RAS mutations and clinical responses to gefitinib. EGFR mutations were detected in 16 (31\%) of the 52 FFPE samples (Table II). Ten samples had EGFR exon 21 L858R point mutations, 5 were exon 19 deletions and 1 had L858R and exon 20 T790M point mutations. K-RAS mutations were detected in 5 (10\%) of 52 FFPE samples (Table II). Four samples had $K-R A S$ codon 12 point mutations and the other sample was a codon 61 point mutation. Among these 52 NSCLC patients, there were 14 patients treated with gefitinib. The treatment outcomes indicated that there were 5 patients with PR, 8 with SD and 1 with PD. Four of the 5 patients with PR had exon 21 L858R point mutations and the other had exon 19 deletions. However, 1 patient with PD also had EGFR mutations. Among the 8 patients with SD, 5 had 


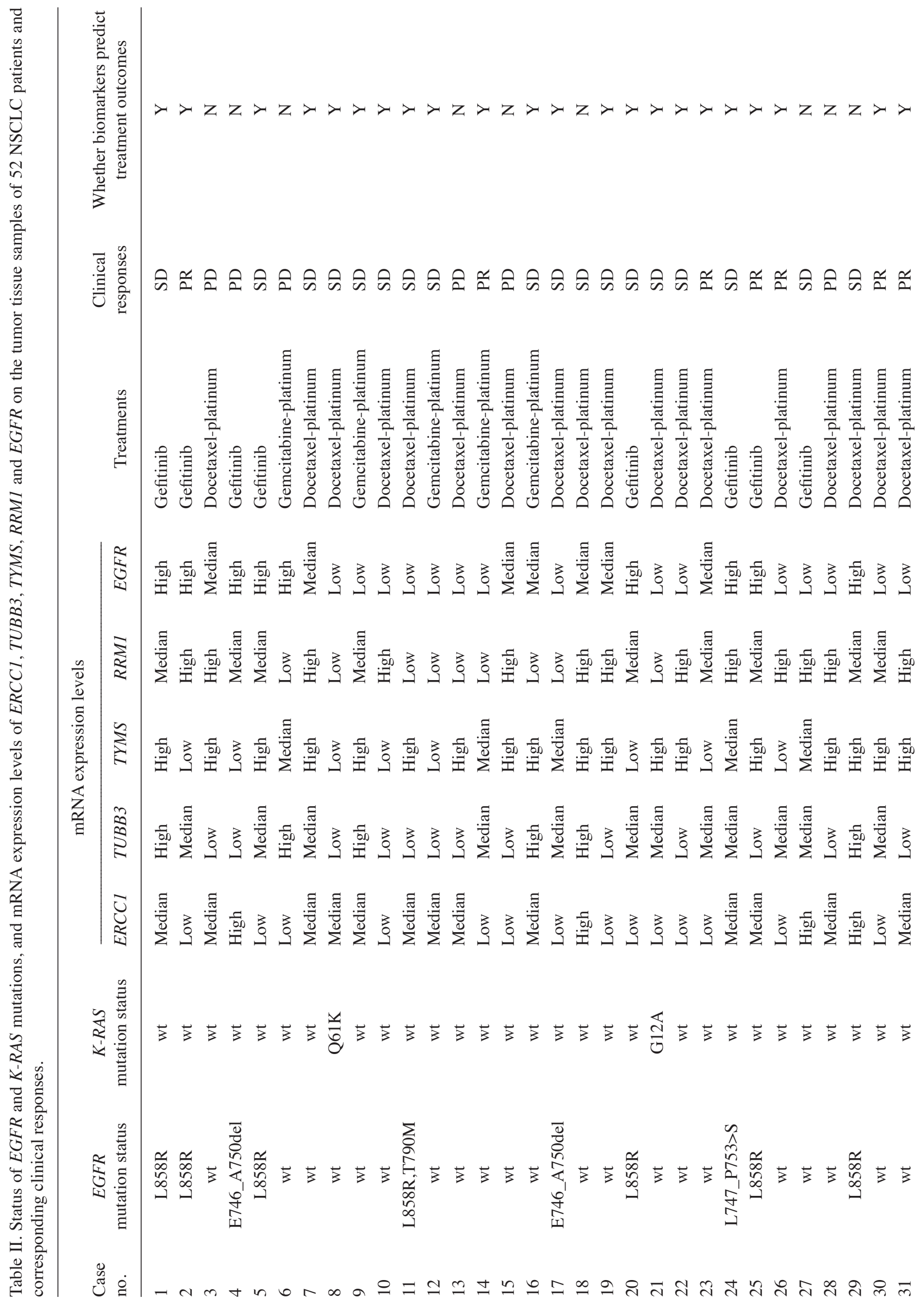




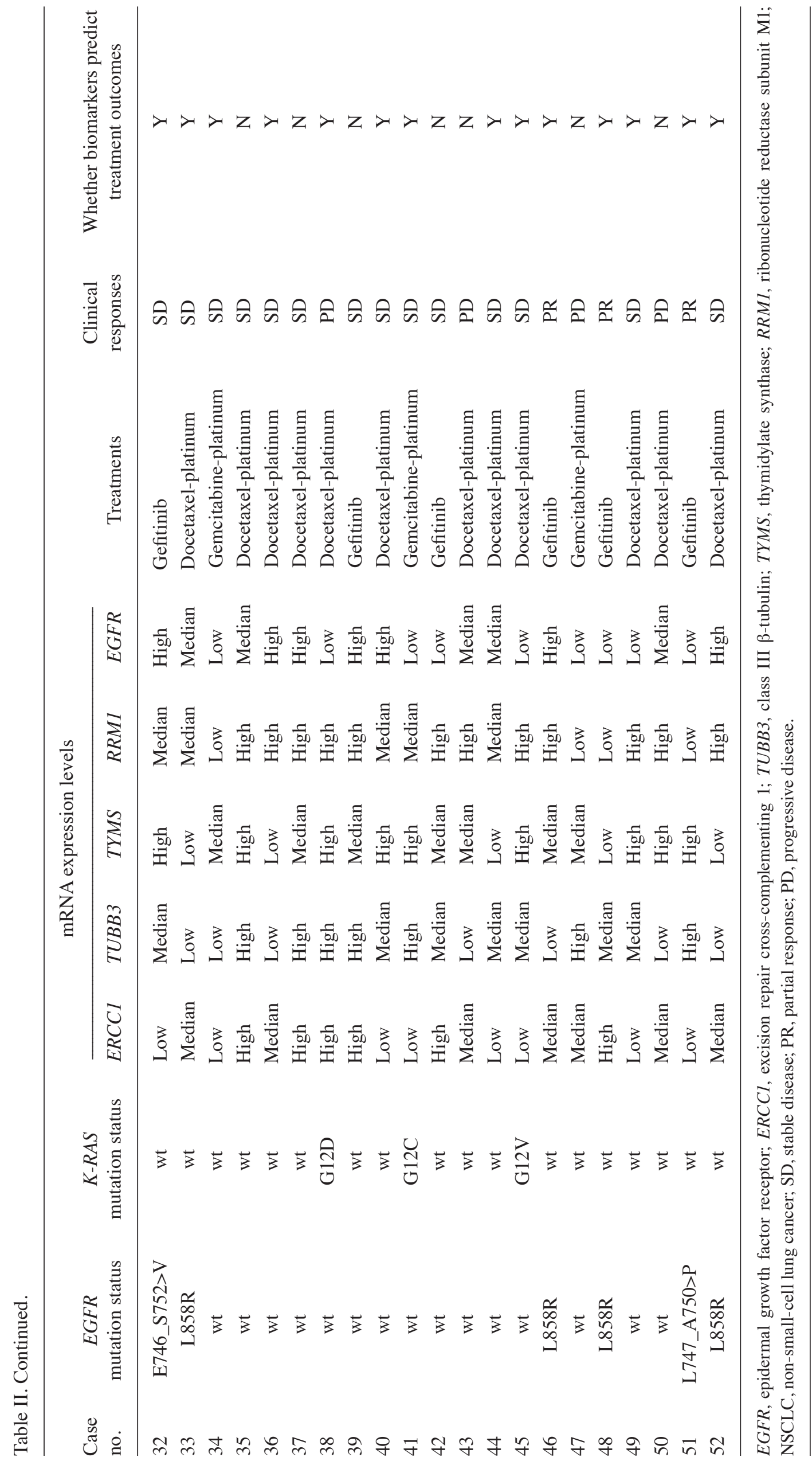


Table III. Summary of ERCC1, RRM1, TUBB3, TYMS and EGFR mRNA expression levels from 52 NSCLC patients.

\begin{tabular}{llllll}
\hline & \multicolumn{5}{c}{ No. of patients (\%) } \\
\cline { 2 - 6 } mRNA expression levels & ERCC1 & TUBB3 & TYMS & RRMI & EGFR \\
\hline High & $10(19)$ & $13(25)$ & $27(52)$ & $25(48)$ & $16(31)$ \\
Median & $20(38)$ & $19(37)$ & $12(23)$ & $14(27)$ & $12(23)$ \\
Low & $22(43)$ & $20(38)$ & $13(25)$ & $13(25)$ & $24(46)$ \\
\hline
\end{tabular}

ERCC1, excision repair cross-complementing 1; RRM1, ribonucleotide reductase subunit M1; TUBB3, class III $\beta$-tubulin; TYMS, thymidylate synthase; $E G F R$, epidermal growth factor receptor; NSCLC, non-small-cell lung cancer.

EGFR exon 21 point mutations or exon 19 deletions and 3 had no mutations. This result indicated that the EGFR mutation status was associated with the efficacy of gefitinib. Among the 14 patients treated with gefitinib, the EGFR mutation status to predict the treatment outcomes could be used in 10 (71.4\%).

mRNA expression levels of ERCC1, RRM1, TUBB3, TYMS and EGFR and clinical responses to chemotherapy. The summary of ERCC1,RRM1,TUBB3,TYMS and EGFR mRNA expression levels is shown in Table III. Among these 52 NSCLC patients, the majority of patients had low (43\%) ERCC1, low TUBB3 (38\%), high TYMS (52\%), high RRM1 (48\%) and low EGFR (46\%) expression. When the patients had low or median ERCCI expression and low or median TUBB3 expression, they were suggested for docetaxel-platinum based chemotherapy treatment. When the patients had low or median ERCCl expression and low or median $R R M 1$ expression, they were suggested for gemcitabine-platinum based chemotherapy treatment.

There were 30 patients treated with docetaxel-platinum and 8 treated with gemcitabine-platinum (Table II). The treatment outcomes indicated that in the docetaxel-platinum treatment group, there were 4 patients with PR, 19 with SD and 7 with PD (Table II). The 4 patients with PR had low or median ERCCl expression and low or median TUBB3 expression. Fifteen of the 19 patients with SD had low or median $E R C C 1$ expression and low or median $T U B B 3$ expression, but 4 of these had high ERCC1 and TUBB3 expression. One of 7 patients with PD had high ERCC1 and TUBB3 expression, however, 6 of these had low or median ERCC1 expression and low or median TUBB3 expression. In the gemcitabine-platinum treatment group, there was 1 patient with PR, 5 with SD and 2 with PD (Table II). The patient with PR had low ERCCl and RRM1 expression. The 5 patients with SD had low or median ERCCl expression and low or median RRMI expression; however, the 2 patients with PD also had low or median ERCCl expression and low or median RRMI expression. Among these 38 patients treated with docetaxel-platinum or gemcitabine-platinum based chemotherapy, the mRNA expression levels of ERCC1 and TUBB3, or ERCC1 and RRM1, of 26 cases could be used to predict the treatment outcomes of chemotherapy $(68.4 \%)$ (Table II).

Correlation between EGFR and K-RAS mutations and ERCC1, RRM1, TUBB3, TYMS and EGFR mRNA expression. The mRNA expression levels of ERCC1, RRM1, TUBB3,
TYMS and EGFR, and the mutation status of EGFR and $K$-RAS were compared. Correlations were observed between the status of EGFR mutations and the mRNA expression levels of EGFR $(\mathrm{P}=0.001, \mathrm{r}=0.437)$. The patients that had $E G F R$ exon 21 L858R point mutations or exon 19 deletions were more likely to have high EGFR expression. No correlation was identified between the other genes.

\section{Discussion}

Chemotherapy has been the traditional treatment for the management of NSCLC. However, the resistance to chemotherapy leads to unsatisfactory treatment outcomes and prognosis. The discovery of molecular markers associated with the clinical responses to chemotherapy and introduction of targeted therapy have refined this traditional treatment for NSCLC. This has led to the concept of personalized medicines. In the present study, efforts in personalizing delivery of care based on the status of EGFR and $K-R A S$ mutations and mRNA expression levels of ERCC1, TUBB3, TYMS, RRM1 and EGFR in choosing appropriate treatments for patients with NSCLC were discussed.

In the present study, gefitinib was chosen as the first-line treatment when the patients were carrying mutations within the tyrosine kinase domain of EGFR, such as mutations in EGFR exon 18, 19 and 21, and no mutations were identified in $K-R A S$ codon 12,13 or 61 . Docetaxel-platinum or gemcitabine-platinum based chemotherapy was chosen as the first-line treatment when the patients had low or median ERCC1/TUBB3 expression, or low or median ERCC1/RRM1 expression, respectively. Although the mRNA expression level of TYMS was analyzed, no fluorouracil-based chemotherapy had been administered. The majority of the patients with low or median TYMS expression also had low or median ERCCI expression or EGFR exon 19 mutations, therefore, platinum-based chemotherapy or gefitinib was administered for treatment. Three patients with wild-type EGFR were treated with gefitinib, as these patients were not suitable for platinum-based chemotherapy and they chose to undergo the EGFR-targeted therapy.

Mutations in EGFR and $K-R A S$ were detected in $16(31 \%)$ and $5(10 \%)$ of the 52 FFPE samples, respectively. One sample had the EGFR exon $21 \mathrm{~L} 858 \mathrm{R}$ and exon 20 T790M point mutations and no samples were identified to have both $E G F R$ and $K-R A S$ mutations. Statistical analysis indicated that the patients that had $E G F R$ exon 21 L858R point mutations or 
exon 19 deletions were more likely to have high EGFR expression. EGFR exon 21 L858R point mutations, exon 19 deletions or high $E G F R$ expression suggested that the patients were sensitive to the gefitinib treatment.

Among these 52 NSCLC patients, there were 14 patients treated with gefitinib and a statistically significant association was revealed between the EGFR mutation status and treatment outcomes of gefitinib in 10 cases $(71.4 \%)$. These findings are consistent with previous studies (18-20). In the other 4 cases, 1 male patient with PD had EGFR exon 19 deletions. This patient was $>70$ years old and had a smoking history of $>20$ years. It is possible that the advanced age and long smoking history of the patient affected the treatment outcomes. Three patients with SD had no EGFR or K-RAS mutations. There were 38 patients treated with chemotherapy; 30 were treated with docetaxel-platinum and 8 with gemcitabine-platinum. The mRNA expression levels of ERCC1 and TUBB3, or $E R C C 1$ and $R R M 1$ could be used in 26 cases to predict the treatment outcomes of chemotherapy $(68.4 \%)$. The clinical response rate of personalized medicine is more efficient than that of the traditional treatments (68.4 vs. $20-40 \%)(21)$.

In conclusion, although the sample size in the study was small, the findings indicated that the mutation status of $E G F R$, as well as the mRNA expression levels of ERCC1, TUBB3 and $R R M 1$, could be used as predictors of response to gefitinib or chemotherapy.

\section{Acknowledgements}

The authors would like to thank SurExam Bio-Tech Co., Ltd., for their technical support to the present study.

\section{References}

1. Siegel R, Naishadham D and Jemal A: Cancer statistics, 2012. CA Cancer J Clin 62: 10-29, 2012.

2. Jazieh AR, Bamefleh H, Demirkazik A, et al: Modification and implementation of NCCN guidelines on non-small cell lung cancer in the Middle East and North Africa region. J Natl Compr Canc Netw 8 (Suppl 3): S16-S21, 2010.

3. Lynch TJ, Bell DW, Sordella R, Gurubhagavatula S, Okimoto RA, Brannigan BW, Harris PL, Haserlat SM, Supko JG, Haluska FG, et al: Activating mutations in the epidermal growth factor receptor underlying responsiveness of non-small-cell lung cancer to gefitinib. N Engl J Med 350: 2129-2139, 2004.

4. Taron M, Ichinose Y, Rosell R, Mok T, Massuti B, Zamora L, Mate JL, Manegold C, Ono M, Queralt C, et al: Activating mutations in the tyrosine kinase domain of the epidermal growth factor receptor are associated with improved survival in gefitinib-treated chemorefractory lung adenocarcinomas. Clin Cancer Res 11: 5878-5885, 2005.

5. Gazdar AF and Minna JD: Inhibition of EGFR signaling: All mutations are not created equal. PLoS Med 2: e377, 2005.
6. Hirsch FR: EGFR: a prognostic and/or a predictive marker? J Thorac Oncol 1: 395-397, 2006.

7. Dacic S, Flanagan M, Cieply K, Ramalingam S, Luketich J, Belani $C$ and Yousem SA: Significance of EGFR protein expression and gene amplification in non-small cell lung carcinoma. Am J Clin Pathol 125: 860-865, 2006.

8. Downward J: Targeting RAS signalling pathways in cancer therapy. Nat Rev Cancer 3: 11-22, 2003.

9. Pao W, Wang TY, Riely GJ, Miller VA, Pan Q, Ladanyi M, Zakowski MF, Heelan RT, Kris MG and Varmus HE: KRAS mutations and primary resistance of lung adenocarcinomas to gefitinib or erlotinib. PLoS Med 2: e17, 2005.

10. Li J, Li ZN, Yu LC, Bao QL, Wu JR, Shi SB and Li XQ: Association of expression of MRP1, BCRP, LRP and ERCC1 with outcome of patients with locally advanced non-small cell lung cancer who received neoadjuvant chemotherapy. Lung Cancer 69: 116-122, 2010.

11. Azuma K, Sasada T, Kawahara A, Takamori S, Hattori S, Ikeda J, Itoh K, Yamada A, Kage M, Kuwano M, et al: Expression of ERCC1 and class III beta-tubulin in non-small cell lung cancer patients treated with carboplatin and paclitaxel. Lung Cancer 64: 326-333, 2009.

12. Burkhart CA, Kavallaris $M$ and Band Horwitz S: The role of beta-tubulin isotypes in resistance to antimitotic drugs. Biochim Biophys Acta 1471: O1-O9, 2001.

13. Bepler G, Sommers KE, Cantor A, Li X, Sharma A, Williams C, Chiappori A, Haura E, Antonia S, Tanvetyanon T, et al: Clinical efficacy and predictive molecular markers of neoadjuvant gemcitabine and pemetrexed in resectable non-small cell lung cancer. J Thorac Oncol 3: 1112-1118, 2008.

14. Rosell R, Danenberg KD, Alberola V, Bepler G, Sanchez JJ, Camps C, Provencio M, Isla D, Taron M, Diz P, et al; Spanish Lung Cancer Group: Ribonucleotide reductase messenger RNA expression and survival in gemcitabine/cisplatin-treated advanced non-small cell lung cancer patients. Clin Cancer Res 10: 1318-1325, 2004.

15. Travis W, Colby TV and Corrin B: Histologic Typing of Tumors of Lung and Pleura: World Health Organization International Classification of Tumors. 3rd edition. Springer Verlag, New York, NY, 1999.

16. Li G, Luo X, He J, Zhu Z, Yu G, Qin H, Zeng T, Liu Z, Wu S, $\mathrm{Xu}$ J,et al: A novel liquidchip platform for simultaneous detection of 70 alleles of DNA somatic mutations on EGFR, KRAS, BRAF and PIK3CA from formalin-fixed and paraffin-embedded slides containing tumor tissue. Clin Chem Lab Med 49: 191-195, 2011.

17. Wang Y, Liu ZD, Zhao LM, Du CX, Xi XM, Mi YL, Peng Y, Li WG, Cheng F, Zhang XR, et al: Individualized treatment of NSCLC: From research to clinical practice. Neoplasma 60: 538-545, 2013.

18. Sharma SV, Bell DW, Settleman J and Haber DA: Epidermal growth factor receptor mutations in lung cancer. Nat Rev Cancer 7: 169-181, 2007.

19. Mitsudomi T and Yatabe Y: Mutations of the epidermal growth factor receptor gene and related genes as determinants of epidermal growth factor receptor tyrosine kinase inhibitors sensitivity in lung cancer. Cancer Sci 98: 1817-1824, 2007.

20. Hirsch FR, Varella-Garcia M, Bunn PA Jr, Franklin WA, Dziadziuszko R, Thatcher N, Chang A, Parikh P, Pereira JR, Ciuleanu T, et al: Molecular predictors of outcome with gefitinib in a phase III placebo-controlled study in advanced non-small-cell lung cancer. J Clin Oncol 24: 5034-5042, 2006.

21. Vadakara J and Borghaei H: Personalized medicine and treatment approaches in non-small-cell lung carcinoma. Pharmgenomics Pers Med 5: 113-123, 2012. 